ORIGINAL ResEARCH ARTICLE

\title{
Advances in Atom Transfer Radical Polymerization of Modified Grain
}

\author{
Changqing Cui,Shaofeng Feng,Liqun Zhu \\ School of Materials Science and Engineering, Chengdu Jiaotong University, Sichuan, China
}

\begin{abstract}
Atom transfer radical polymerization (ATRP) is a kind of controllable reactive radical polymerization method with potential application value. The modification of graphene oxide (GO) by ATRP reaction can effectively control various graft polymer molecules Chain length and graft density, giving GO different functionality, such as good solvent dispersibility, environmental sensitive stimulus responsiveness, biocompatibility, and the like. In this paper, ATRP reaction and GO surface non-covalent bonding ATRP polymer molecular chain were directly initiated from GO surface immobilization initiator. The ATRP reaction modified GO was reviewed, and the process conditions and research methods of ATRP modification reaction were summarized, as well as pointed out the functional characteristics and application prospect of GO functionalized composites.
\end{abstract}

KEYWORDS: Keywords:Watom transfer radical polymerization; Graphene oxide; Modification

PCrude graphene oxide (GO) is a derivative of graphene, which is obtained by chemical oxidation of natural graphite and then ultrasonic stripping [1-3]. Although the chemical oxidation process of GO has destroyed the highly conjugated structure of graphene, it still maintains special surface properties and lamellar structure, and a large number of oxygencontaining functional groups such as hydroxyl group, carboxyl group, Epoxy group [4-6], which is conducive to further functional modification. The functional modification of GO is an important means to expand its application range. The ideal modification method is to selectively modify the surface properties of GO and the different properties of oxygencontaining functional groups by various chemical reactions. Atom transfer radical polymerization (ATRP) is one of the most important and effective methods of reactive radical polymerization. It has the characteristics of fast reaction speed, mild reaction condition and wide application range. The molecular design ability is strong, and it is beyond comparison by other existing active Polymerization methods [7,8]. The ATRP modification method for GO consists of two methods: one is to fix the ATRP initiator on the GO layer, initiate the monomer polymerization, graft the functional polymer molecular chain; the second is through the ATRP reaction to obtain the functional polymer Long chain molecules, and then non-covalently bonded to the GO layer. In this paper, the ATRP reaction modified GO studies are reviewed. The results of the present study show that the two ATRP functionalized modification methods can change the surface polarity of GO and the dispersibility in the solution, GO has a wide variety of special features that make it more widely used in the fields of light, electricity, magnetic and biosensors.

\section{ATRP functional modification was carried out using GO surface oxygen- containing functional group immobilized initiator}

TDue to the reactivity of the oxygen-containing functional groups on the surface and edge of the GO layer, it can be a reactive site for the immobilized ATRP initiator, and the presence of the oxygen-containing functional groups allows the Au colloid to form an electrostatic repulsion on the surface, and significantly improves dispersion stability of the GO in water, dimethylformamide (DMF), N-methylpyrrolidone (NMP), tetrahydrofuran (THF), ethylene glycol and other organic solvents[9], which for the solution environment to achieve GO ATRP functional modification possible.

Copyright (C) 2017 -. This is an Open Access article distributed under the terms of the Creative Commons Attribution-NonCommercial 4.0 International License (http://creativecommons.org/licenses/by-nc/4.0/), permitting all non-commercial use, distribution, and reproduction in any medium, provided the original work is properly cited. 


\subsection{Utilise GO surface hydroxyl as a reactive point}

The surface of the GO nanosheet is rich in hydroxyl functional groups. The ATRP initiator can be immobilized on the GO surface by the reaction site provided by the hydroxyl group. The grafted functional polymer can be initiated by covalent bonding for branched modified GO nanocomposites.

Lee et al. [10] proposed a functional modification method for the growth of polymers directly on the GO surface using the ATRP method, i.e., the esterification reaction of isobutyryl bromide with the hydroxyl groups on the GO surface, covalently bonding the bromide initiator (St), methyl methacrylate (MMA) or butyl acrylate (BA) polymers (Fig. 1). The improved solubility of GO after functional modification makes the chemical characterization and modification process can be carried out in a single homogeneous system, and the increase of solubility and dispersibility also enhances the potential application of GO in polymer composites. In addition, the ATRP reaction can also be used to polymerize the more complex monomer modification GO, which provides a quick way to adjust the physical properties, thermal and electrical properties of GO and its related compounds.
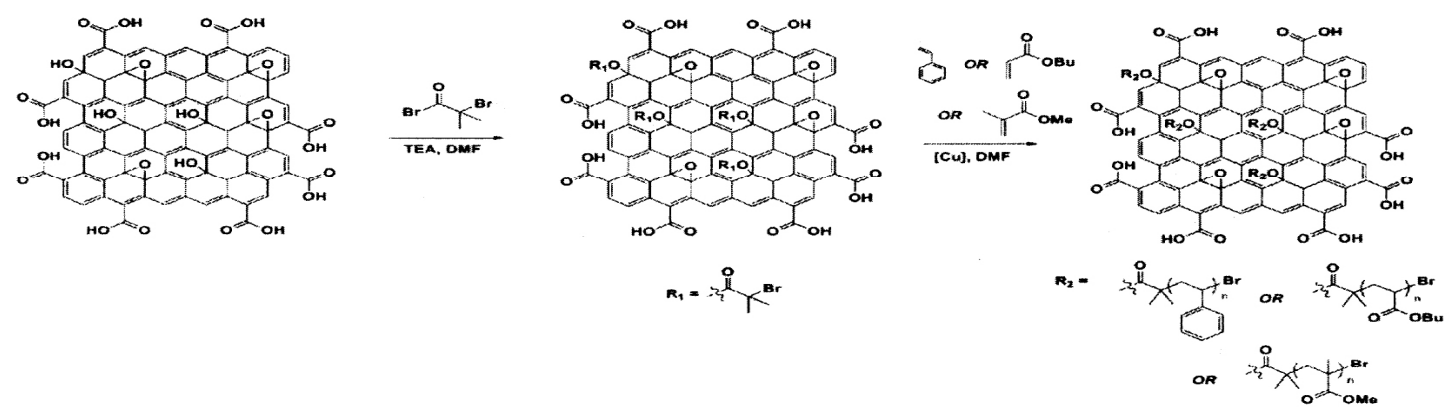

Fig. 1

Hossein et al. [11] oxidized peeling of graphite to produce GO containing a large amount of hydroxyl groups on the surface. First, the surface of GO was modified by the coupling reaction of 3-aminopropyltriethoxysilane (APTES) with hydroxyl groups, and then the initiator of ATRP reaction 2-Bromo-2-methylpropionyl bromide (BiBB) was reacted with APTES so that the initiator was immobilized on the GO surface to initiate the polymerization of St to obtain polystyrene (PSt) functionalized GO with different grafting densities Layer. It was found that the high grafting density expanded the GO layer spacing more uniformly, and the GO nanosheets uniformly coated with PSt were separated from each other to form a monolayer structure in the stripped state. Due to the influence of the oxidation reaction and the functionalization process, the GO layer showed obvious folds and bends.

Mr Lik et al. [12] showed the ATRP initiator was covalently bound to the hydroxyl group on the GO surface and the grafted poly (methyl methacrylate) (PGMA) grafted GO was prepared by surface-initiated ATRP reaction as a dispersion corresponding to the silicone oil alternating current suspension. Studies showed that the method of modifying ATRP grafted polymer on GO surface able to obtained by one-step reaction, which was compatible with carrier solution and had good conductivity. GO was reduced during ATRP polymerization, and improved conductivity. This modification method not only can control the reduction degree of GO, but also make GO produce fine-tuning electrical response, which is effectively applied to electrorheological suspension, compared with ordinary graphene-based electrorheological suspension, the polarized modified GO enhances the electrorheological properties of such smart materials.

Li et al. [13] used 4-chloromethylphenylsilane to couple with the hydroxyl groups on the GO nanosheet. First, the ATRP initiator was immobilized on the surface of GO, and then ATRP was initiated on the surface to obtain surface grafted hydrophobic polyacrylic acid Tert-butyl ester (PtBA) polymer brush GO-g-PtBA nanocomposites. This enhances the solubility and dispersibility of GO in organic solvents. The functionalized modified GO is easy to dissolve in toluene and can be incorporated into the electroactive 3-hexylthiophene (P3HT) film. The resulting composite membrane exhibits bistable electrical conductance and nonvolatile electron storage effect. In addition, GO-g-PAAc (PAAc, polyacrylic acid) nanosheets were produced by the hydrolysis of GO-g-PtBA under the action of hydrolysis, so that the gold nanoparticles modified GO-g-PAAc could be prepared in the mixed aqueous dispersion nano-film.

Layek et al. [14] prepared GO by the Hummers method and then immobilized the ATRP initiator 2-bromoisobutyryl bromide (2-BIB) on the GO surface with hydroxyl groups as active sites, initiating grafting of polymethylmethacrylate (PMMA) and then reduced by hydrazine hydrate to obtain PMMA-functionalized graphene (MG) nanosheets as a nucleating agent in PVDF to prepare piezoelectric, electrically conductive, biocompatible high performance composites, suitable for different application needs. MG nanosheets increase the $\alpha$-crystal phase of PVDF and increase the $\beta$-crystal phase. With the increase of graphene content, the distribution of graphene nanosheets changes from parallel orientation to random orientation. The results show that the heat stability of the composites increased significantly, the storage modulus, tensile strength and elastic modulus were improved to some extent, and the composite material showed the 
electrodialysis extreme value at 3.8\% MG content. It is worth mentioning that the use of ATRP grafted modified GO nanocomposites can be achieved without the need for additional reduction reaction steps, and Ilcíková et al. [15] pointed out that the surface-initiated ATRP modification method can be carried out under one-step reaction conditions at the same time, the in situ reduction and modification of GO were completed, and the conductive graphene structure was partially recovered to obtain conductive hybrid composites, which simplified the functionalized modification process of GO.

\subsection{Utilize GO surface epoxy as a reactive point}

GO surface of the epoxy group with some amino groups with small molecules in the nucleophilic reaction, can go through a simple open-loop reaction to achieve GO modification. The ATRP initiator was immobilized on the GO surface using the epoxy group as the reactive site, which in turn induced the molecular chain of the graft polymer, thus realizing the functional modification of GO.

Qi et al. [16] combined ATRP initiator, N-(2-aminoethyl)-2-bromo-2-methylpropanamide (NABM), was synthesized by the reaction of the primary amine groups on the NABM with the epoxy groups on the GO surface Nucleophilic ring-opening reaction, the GOMA-g-GO nanocomposite material (Fig.2), which is functionalized by grafting PMMA, was prepared and used as a soluble protective coating. The results show that the PMMA-g-GO coating can effectively prevent the charge transfer between the metal-electrolyte interfaces and prevent the copper substrate from being stronger and prevent copper substrate from corrosion under salt conditions.
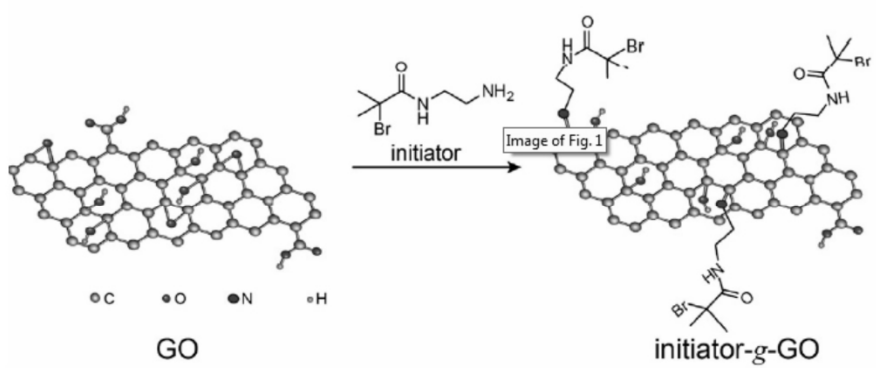

Fig. 2

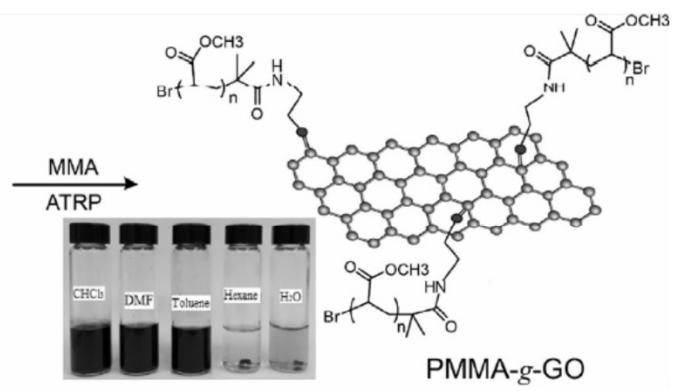

PMMA-g-GO

Hossein [17] reported that a bifunctional modifier containing ATRP initiating group and an amine group was synthesized by coupling reaction of ethylenediamine with $\alpha$-isobutyryl bromide N- (2-aminoethyl) -2-bromide (GO-Br), which was modified by the open-loop reaction of the epoxy group on the GO surface, was coupled with the amine group of $\mathrm{OBr}$ to obtain the oxidized graphene $(\mathrm{GO}-\mathrm{Br})$ with different density $\mathrm{OBr}$, and then the ATRP Reaction, preparation of different graft density of long chain PSt. After oxidation and functionalization, GO nanosheets exhibit large amounts of folds and folds, and the presence of PSt makes the GO nanosheet opaque.

\subsection{Utilize GO edge carboxyl as a reactive point}

By utilizing carboxyl group at the edge of GO for functional modification, Thionyl chloride (SOC12), 1(3-dimethylaminopropyl) -3-ethylcarbodiimide hydrochloride (EDC), N-dimethylformamide N, N'-tetramethyluronium hexafluorophosphate (HATU), N'-dicyclohexylcarbodiimide (DCC) or 2- (7-azobenzotriazole) types of activator activates the carboxyl group and then reacts with the amine or hydroxyl group through a covalent cross-linking reaction on the GO surface.

Combined with functional groups to form the corresponding amine or ester [18]. Through these reactions, the ATRP initiator can be immobilized on the GO layer, triggering the molecular chain of the graft polymer, and functionalizing the GO.

Masoumeh et al. [19] prepared GO by an improved Hummers method. The ATRP initiator was immobilized on the GO surface by two-step modification, i.e., the carboxyl group at the GO edge was activated with thionyl chloride to chlorinated acyl groups to increase the carbonyl group, then reacted with ethylenediamine and triethylamine to obtain GO-NH2 powder, and then through the amine group and 2-bromoisobutyryl bromide coupling reaction to get GO-Br, with ATRP reaction successfully grafted in the GO surface Polyhydroxyethyl methacrylate (PHEMA). It was found that the grafted PHEMA GO showed a response to $\mathrm{pH}$ and temperature stimulation.

Gil et al. [20] activated the carboxyl groups on the GO layer to an acid chloride group and treated with ethylene glycol to give a hydroxyl-rich graphene sheet. The ATRP initiator BiBB was then immobilized and successfully passed through the ATRP polymerization. Grafted PMMA molecular chain, modified PMMAg-GO nanocomposites easily 
dispersed in organic solvents. The researchers proposed the use of ATRP functional modification method, only need to replace the polymerization monomer can be grafted in the GO surface of different types of polymer molecular chain.

Kavitha et al. [21] prepared by the improved Hummers method, first GO, EDC as an activator initiated GO edge of the carboxyl and propylene diamine reaction to form amide bond, so that GO modified amine, through the amine and $\mathrm{BiBB}$ reaction to fix ATRP initiators were then grafted onto GO with different relative molecular mass of polyvinyl caprolactam (PVCL). The biocompatible PVCL makes the GO layer highly dispersed and stabilized in water and physiological solutions. The PVCL functionalized GO is used as a carrier to transport water insoluble anticancer drugs (CPT) into cancer cells and the drug can be released by $\mathrm{pH}$ and temperature control. The results show that this new composite material with biocompatibility, drug delivery and stimulating response has many prospects in the field of biology and medicine.

In addition to the activation of the carboxyl group at the GO edge, it is also possible to use an amphoteric modifying agent directly with the carboxyl group at the GO edge for amidation or esterification, and the initiator is immobilized on the GO sheet. Yang et al. [22] prepared the stripped GO by the Staudenmaier method, amidated by the carboxylation of the GO edge and the bifunctional coupling agent, introduced the amine group on the GO surface, and then reacted with the ATRP initiator BiBB, (PDMAEMA) molecular chain on the surface of GO-grafted poly (meth) acrylate by in situ ATRP polymerization, and the active carboxylic acid group Of the polyethylene glycol dimethacrylate-methacrylic acid copolymer (EGDMA-co-MAA) particles were deposited on the GO layer (Fig. 3). The results show that the dispersion of the GO layer after modification of the polymer in the solvent is significantly improved. The method of preparing the functionalized nanocomposite by modifying the GO directly will greatly promote the development and application of the GO material.

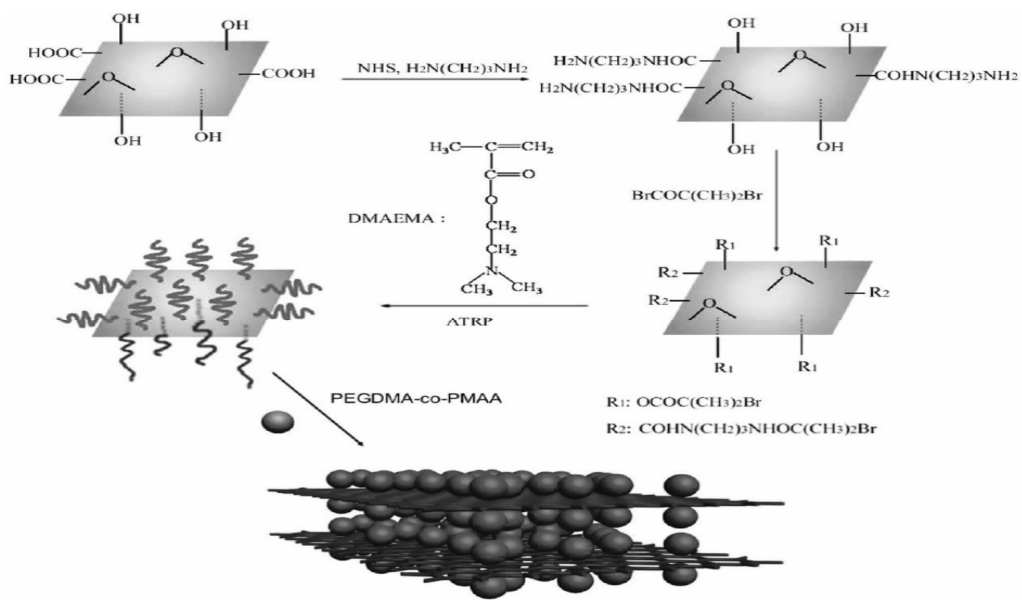

Fig. 3

Hossein et al. [23] synthesized a bifunctional modifier containing a double bond and a hydroxyl group, and the modifying agent was attached to the GO layer by the esterification between the hydroxyl group of the modifier and the carboxyl group at the GO edge The modified GO and St monomers participate in the ATRP polymerization reaction, and finally the GO/PSt nanocomposites are obtained. The existence of PSt increases the pitch of the GO layer. The higher the PSt graft density in the composites, the higher the glass transition temperature In the process of oxidation and functionalization, the GO layer is bent from smooth and wrinkled, and the transparency of the nanosheets decreases.

\section{The GO carbon skeleton fixed initiator for ATRP functional modification}

The main structure of the graphene is a honeycomb-like two-dimensional planar film composed of a single layer of carbon atoms. The unsaturated $\mathrm{C}=\mathrm{C}$ double bond on the carbon atom of the monatomic layer is modified by some active groups, and the ATRP The initiator is directly immobilized on the surface of the graphene oxide to initiate grafting of the functional polymer.

Peng et al. [24] used the Hummer Offeman method to prepare graphene oxide, which converts the ATRP active material with the $\mathrm{C}=\mathrm{C}$ double bond in the $\mathrm{GO}$ carbon skeleton. This reaction can directly immobilize small and large ATRP initiator molecules, followed by the initiation of the ATRP reaction on the surface, binds the polymer chain to the GO layer and successfully grafts a polymer brush of different architectures, such as linear, V-type and graded layers, on the GO surface ( Fig.4). After the grafted polymer modified GO, its organic compatibility is improved, can be dispersed 
in the organic solvent for more than $80 \mathrm{~d}$. The study explored an effective way to expand the range of polymers used for GO graft modification to produce more polymer/GO composite nanomaterials with different properties.

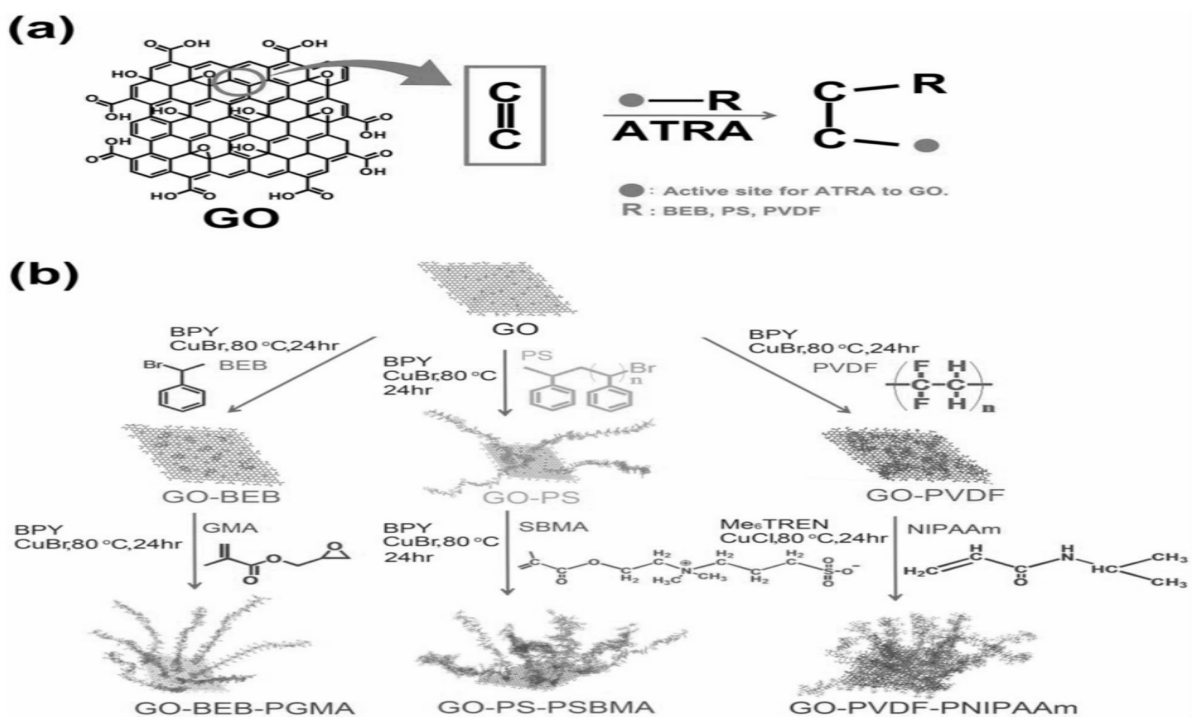

Fig. 4

Bak et al. $[25,26]$ GO were prepared by Brodie method and subjected to thermal reduction to obtain bulk functionalized graphene sheets (FGSs). The FGSs lamellae were first treated with 4-aminophenylethyl alcohol and the hydroxyl functional groups were modified on their carbon skeleton and then reacted with $\alpha$-bromoisobutyryl bromide to immobilize the ATRP initiator. Then, the methylene diethylenetriamine (PMDETA) was grafted on the graphene sheet by surface-initiated ATRP polymerization [25] and poly (butyl acrylate) (PtBA) [26]. It was found that the reversible phase transfer between the aqueous and organic phases of the FGS-PDMAEMA hybrid material can be accomplished by controlling the temperature change by controlling the temperature change of the grafted PMDETA polymer brush, selectively dissolving in water or an organic solvent, while the material wettability can be controlled. (FGS-PAA) was obtained by hydrolysis of tert-butyl group with Pt-BA grafted FGSs. It was proved that the dispersion and agglomeration of FGS-PAA hybrid material in water could be changed by adjusting the $\mathrm{pH}$ of solution state, this controllable dispersion helps to improve the performance of graphene-based hybrid materials, indicating its potential applications in the fields of electronics, energy and sensors.

Fang et al. [27] single layer graphene nanosheets (SLGN), the reaction process of immobilizing ATRP initiator on graphene carbon skeleton was obtained by the addition of sodium dodecylbenzene sulfonate (SDS), and the subsequent ATRP grafting of the PSt molecular chain, the graft density and molecular chain length of the polymer were controlled by adjusting the concentration of the diazo compound and monomer. The results show that the molecular weight distribution of the SLGN-PS sample with high graft density is more uniform than that of the low graft density, and the polymer chain is covalently bonded to the SLGN. The relaxation motion is strongly restricted. The thermal conductivity of SLGN-PSt nanocomposites increases with the increase of the thermal conductivity of the graphene composites when the mass fraction of SLGN is only $2.0 \%$.

\section{The combination of ATRP polymer and GO noncovalent bond functional modification}

The non-covalent bond moiety is modified by the ATRP reaction to obtain the functional polymer long chain molecule, and then the non-covalent bond is used to bond the polymer molecular chain to the GO layer. The functionalized modification method resulting polymer-GO nanocomposites have excellent properties.

$\mathrm{Xu}$ et al. [28] first used the bifunctional ATRP initiator PBI-Br to initiate the polymerization of acetone glycidyl acrylate (SA) monomer, and successfully prepared a water-soluble diimide-containing polypropylene triacrylate (PBIPGA). This is followed by using the improved Hummers method for GO synthesis, and subjected by annealing to achieve reduced GO (RGO). Then, the pre-synthesized PBIPGA and RGO nanosheets were self - assembled by $\pi-\pi$ stacking interaction, and finally RGOPBIPGA (Fig.5). It has been found that RGO-PBIPGA exhibits good dispersibility in water while showing very low cytotoxicity to $3 \mathrm{~T} 3$ fibroblasts, so the composite can be used for biomedical applications such as cell Imaging and intracellular drug delivery. 


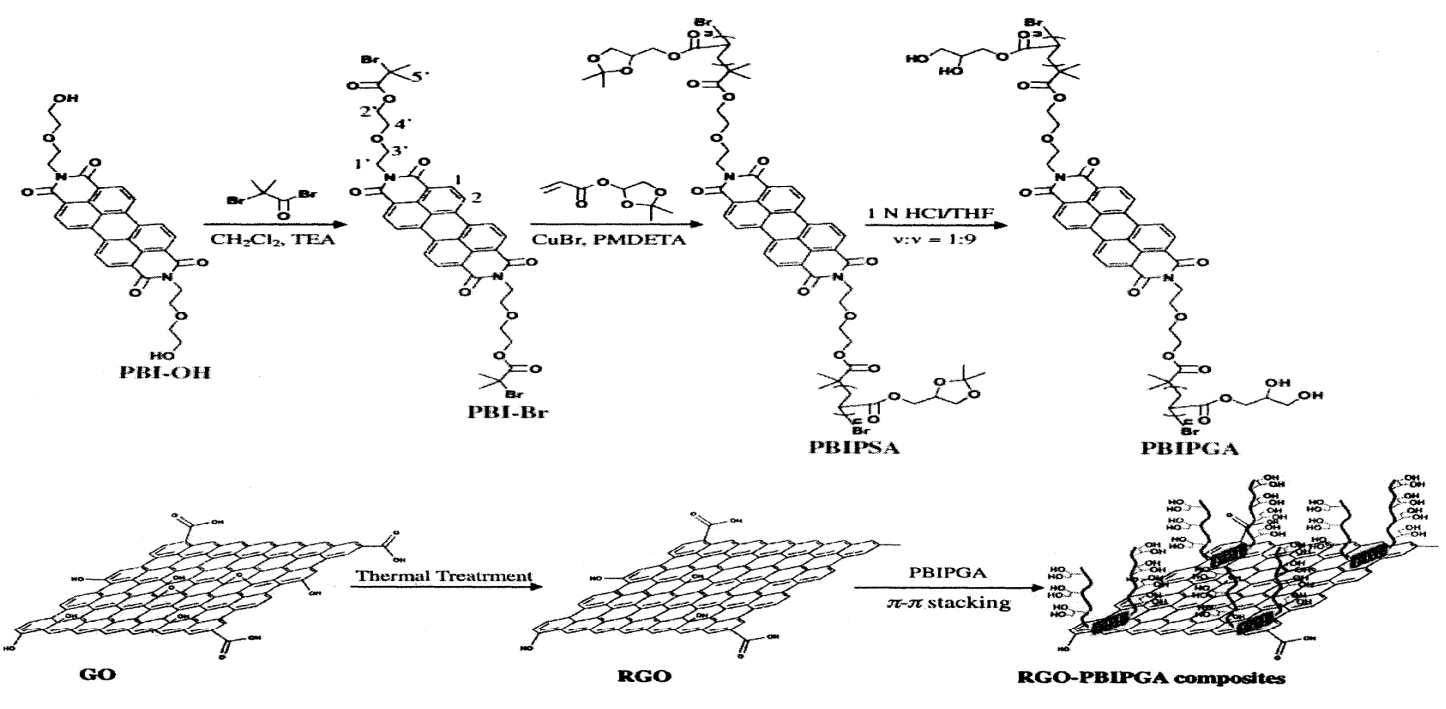

Fig. 5

Song et al. [29] synthesized pyrene molecular functionalized polymethylmethacrylate-polydimethylsiloxane block copolymer (Py-PMMAb-PDMS) by electron transfer atom transfer radical polymerization (ARGET ATRP) (Py-PMMAb-PDMS) microparticles were prepared by the interaction of the pyrene molecules in the copolymer with the $\pi-\pi$ of the GO carbon layer and dispersed in the solution by the solution mixing method. It was found that GO @, Py-PMMA-bPDMS achieves good dispersion effect when the mass fraction of PMMA is $0.05 \%$ to $1.0 \%$, and the lower content of GO @ Py-PMMA-b- The addition of PDMS can acheive the enhancement and toughening of PMMA at the same time, and the optical properties and thermal stability of PMMA nanocomposites are also improved. The results show that this noncovalent bonding functionalized GO @ Py-PMMA-b-PDMS is an ideal filler for the preparation of high performance PMMA nanocomposites, which can impart stable mechanical properties, optical and thermal properties.

\section{Conclusion}

ATRP plays an important role in the various functionalization methods of GO [30], which is an important topic in the field of scientific research in recent years. GO research and application of modified to provide a new way of thinking. In general, there are two main ways to achieve the functional modification of GO by using ATRP reaction: First, the ATRP reaction is directly initiated on the GO surface. Second, the ATRP polymer molecules are covalently bonded to the GO surface chain. The first approach forms a strong irreversible covalent bond between GO and the polymer molecular chain, and overcomes the large steric hindrance effect of the GO nanosheet, which is beneficial to control and improve the graft density of the polymer. The GO/polymer composites have good controllability in terms of physical, mechanical and electrical properties, due to the weak noncovalent bond combination that avoids the introduction of sp3 defective sites on the GO surface. In addition, the ATRP modification process can be either a grafting method on the GO surface or a grafting method at the GO edge. This specific stereoselectivity is also very useful for the functionalization of GOspecific regions significant meaning. The modification of the polymer with the ATRP reaction on the GO nanometer lamellae has the characteristics of structural diversity, which will promote the development of GO from the laboratory to become an important component in many technical applications. The future ATRP reaction modified GO The research direction will be more inclined to develop and prepare a composite multi-performance GO/polymer composite material, making it more widely used in nanocomposites, molecular self-assembly, chemical sensors, optoelectronic materials, catalysts and medical drug transport and other fields.

\section{Reference}

1. Brodie B C. On the atomic weight of graphite [J]. Philos. Trans.R. Soc. London, 1859, 149: 249-259.

2. Staudenmaier L. Method for the preparation of graphitic acid [J]. Berichte der Deutschen Chemischen Gesellschaft, 1898 , 31:1481-1487.

3. Hummers W S, Offeman R E. Preparation of graphitic oxide [J].J. Am. Chem. Soc. , 1958, 80: 1339.

4. Mkhoyan K A, Contryman A W, Silcox J, et al. Atomic and electronic Structure of graphene-oxide [J]. Nano Lett. , 2009,9:1058-1063.

5. Gao W, Alemany L B, Ci L J, et al. New insights into the structure and reduction of graphite oxide [J]. Nat. Chem. , 2009,1:403-408

6. Dreyer D R, Park S, Bielawski C W, et al. The chemistry of graphene Oxide [J]. Chem. Soc. Rev., 2010, 39: 228-240. 
7. Krzysztof M, Xia J H. Atom transfer radical polymerization [J] Chem. Rev., 2001, 101: 2921-2990.

8. Matyjaszewski K, Tsarevsky N V. Nanostructured functional Materials prepared by atom transfer radical polymerization [J]. Nat. Chem., 2009,1: 276-288.

9. Paredes J I, Villar-Rodil S, Martínez-Alonso A, et al. Graphene Oxide dispersions in organic solvents [J]. Langmuir, 2008, 24:10560-10564.

10. Lee S H, Daniel R D, An J, et al. Polymer brushes via controlled, Surface-initiated atom transfer radical polymerization (ATRP) from graphene oxide. [J]. Macromol. Rapid Commun ,2010, 31: 281-288.

11. Hossein R M, Vahid H A. In-plane functionalizing graphene Nanolayers with polystyrene by atom radical waste polymerization:Grafting from hydroxyl groups [J]. Polym. Compos.,2014, 35: 386-395.

12. Mrlík M, IlcíkováM, Plach $\square$ T, et al. Graphene oxide reduction During surface-initiated atom transfer radical polymerization Of glycidyl methacrylate: measures electro-responsive properties[J]. Chem. Eng. J. , 2016, 283: 717-720.

13. Li G L, Liu G, Li M, et al. Organo-and water-dispersible Graphene oxide-polymer nanosheets for organic electronic memory and gold nanocomposites [J]. J. Phys. Chem. C, 2010,114:12742-12748.

14. Layek R K, Samanta S, Chatterjee D P, et al. Physical and Mechanical properties of poly (methyl methacrylate) -functionalized Graphene / poly (vinylidine fluoride) nanocomposites: piezoelectric? Polymorph Formation [J]. Polymer, 2010, 51: 5846-5856.

15. IlcíkováM, Mrlík M, Spitalsk $\square Z$, et al. A tertiary amine in Two competing processes: reduction of graphene oxide vs. Catalysis Of atom transfer radical polymerization [J]. RSC Adv., 2015, 5: 3370-3376.

16. Qi K, Sun Y M, Duana H W, et al. A corrosion-protective Coating based on a solution-processable polymer-grafted graphene Oxide nanocomposite [J]. Corros. Sci., 2015, 98: 500-506.

17. Hossein R M. Surface-initiated ATRP of styrene from epoxy Groups of graphene nanolayers: twofold polystyrene chains and various graft densities [J]. RSC Adv. , 2015, 5: 53357-53368.

18. Veca L M, Lu F, Meziani M J, et al. Polymer functionalization and solubilization of carbon nanosheets [J]. Chem. Commun ,2009, 18: 2565-2567.

19. Masoumeh N, Mehdi S K, Mahdi S H. Dual thermo-and pHsensitive Poly (2-hydroxyethyl methacrylate-co-acrylic acid) Grafted Graphene oxide [J]. Colloid Polym. Sci., 2014, 292: 2599-2610.

20. Gil G, Paula A A P M, Ana B T, et al. Graphene oxide modified With PMMA via ATRP as a reinforcement filler [J]. J. Mater. Chem., 2010, 20: 9927-9934.

21. Kavitha T, Kang I K, Park Y S. Poly (N-vinyl caprolactam) grown on nanographene oxide as an effective nanocargo for drug Delivery [J]. Colloids Surf. , B, 2014, 115: 37-45.

22. Yang Y F, Wang J, Zhang J, et al. Exfoliated graphite oxide By PDMAEMA chains and polymer particles [J].Langmuir, 2009, 25: 11808-11814.

23. Hossein R M, Vahid H A, Khezrollah K, et al. Edgefunctionalized Graphene nanoplatelets with polystyrene by atom Transfer radical polymerization Groups [J]. Polym. Int., 2014, 63: 1912-1923.

24. Peng K J, Wang K H, Hsu K Y, et al. Building up polymer architectures on graphene oxide sheet surfaces through sequential Atom transfer radical polymerization [J]. J. Polym. Sci. Part A: Polym. Chem., 2014, 52: 1588-1596.

25. Bak J M, Lee T, Seo E, et al. Thermoresponsive graphene Nanosheets by functionalization with polymer brushes [J]. Polymer, 2012, 53: 316-323.

26. Bak J M, Lee H. PH-tunable aqueous dispersion of graphene Nanocomposites functionalized with poly (acrylic acid) brushes [J]. Polymer, 2012, 53: 4955-4960.

27. Fang M, Wang K G, Lu H B, et al. Single-layer graphene Nanosheets with controlled grafting of polymer chains [J]. J. Mater. Chem., 2010, 20: 1982-1992.

28. Xu L Q, Wang L, Zhang B, et al. Functionalization of reduced Graphene oxide nanosheets via stacking interactions with the fluorescent and water-soluble perylene bisimide-containing polymers[J]. Polymer, 2011, 52: 2376-2383.

29. Song S Q, Wan C Y, Zhang Y. Non-covalent functionalization of graphene oxide by pyrene-block stocks for enhancing Physical properties of poly (methyl methacrylate [J] .RSC Adv. 2015, 5: 79947-79955.

30. Rama K L, Arun K N. A review on production and properties of polymer functionalized graphene [J] Polymer, 2013, 54: 50875103. 